\title{
Occurrence of Foot-and-Mouth Disease Virus Serotypes in Uganda and Tanzania (2003 to 2015): A Review and Implications for Prospective Regional Disease Control
}

\author{
Susan D. Kerfua ${ }^{1,2}$, Moses T. Dhikusooka ${ }^{2}$, Alice L. Mulondo ${ }^{2}$, James Bugeza ${ }^{2}$, Fredrick Kabi ${ }^{2}$, Shirima Gabriel ${ }^{1}$, \\ Lughano Kusiluka ${ }^{3}$, Chrisostom Ayebazibwe $^{4}$, Sarah Cleaveland ${ }^{5} \&$ Daniel T. Haydon ${ }^{5}$ \\ ${ }^{1}$ Nelson Mandela African Institution of Science and Technology, Arusha, Tanzania \\ ${ }^{2}$ National Livestock Resources Research Institute, Wakiso, Uganda \\ ${ }^{3}$ Mzumbe University, Morogoro, Tanzania \\ ${ }^{4}$ Food and Agriculture Organisation, Kampala, Uganda \\ ${ }^{5}$ Institute of Biodiversity, Animal Health and Comparative Medicine, University of Glasgow, United Kingdom \\ Correspondence: Susan D. Kerfua, National Livestock Resources Research Institute, P.O. Box 5704, Kampala, \\ Uganda. Tel: 256-706-392-098. E-mail: kerfuas@gmail.com; kerfuas@nm-aist.ac.tz
}

Received: May 4, 2019

Accepted: July 18, 2019 Online Published: May 15, 2020

doi:10.5539/jas.v12n6p119

URL: https://doi.org/10.5539/jas.v12n6p119

\begin{abstract}
Endemic foot-and-mouth disease (FMD) presents a global economic challenge to the livestock industry. The progressive control pathway for FMD (PCP-FMD) specifies successive steps through which a country/region can reduce FMD virus circulation and impact. These steps are reliant on understanding and obtaining knowledge on FMD epidemiology, to inform development of appropriate disease interventions like vaccination and quarantine programs. Currently, Uganda and Tanzania are in the early stages of the PCP-FMD.

This review was undertaken to determine FMDV serotype distribution in Uganda and Tanzania between 2003 and 2015. The paper also presents the vaccine strains used in both countries for the same period viz avis the circulating topotypes. The review highlights four $(\mathrm{O}, \mathrm{A}$, SAT 1 and SAT 2$)$ and five $(\mathrm{O}, \mathrm{A}$, SAT 1, SAT 2 and SAT 3) serotypes that occurred in Uganda and Tanzania respectively in the thirteen year period. Observations revealed that reported circulating serotypes $\mathrm{O}$ and $\mathrm{A}$ in the two countries belonged to similar topotypes, East African 2 (EA-2) and AFRICA respectively. The SAT 1 viruses in Tanzania belonged to topotype I and differed from the Ugandan SAT 1s that belonged to topotype IV. Similarly, the SAT $2 \mathrm{~s}$ in both countries belonged to different topotypes: IV in Tanzania and I in Uganda. This review additionally, underscores the spatial distribution of FMDV serotypes in Uganda and Tanzania and highlights regions in both countries that had high serotype diversity.

The paper recommends definitive disease diagnoses, molecular serotype characterisation and matched vaccination deployment for improved disease control.
\end{abstract}

Keywords: control, East Africa, epidemiology, foot-and-mouth disease, serotypes

\section{Introduction}

\subsection{Foot-and-Mouth Disease}

Foot-and-mouth disease (FMD) is a highly infectious and economically important livestock disease (James \& Rushton, 2002; Food and Agricultural Organisation [FAO], 2018). The notifiable disease is caused by the foot-and-mouth disease virus (FMDV), a member of Aphthoviridae genus and the family of Picornaviruses (Thompson \& Bastos, 2004). The virus has seven serotypes; O, A, C, SAT 1, SAT 2, SAT 3 and Asia 1 (Jamal \& Belsham, 2013). Foot-and-mouth disease is spread through contact of susceptible non-infected animals with those that are infectious. Fomites such as clothes, cars tyres and farm tools can be important agents for virus transmission (Kitching, 2005). Some studies have documented FMD spread through aerosols and wind currents and, have recorded virus transmission of $250 \mathrm{~km}$ over sea and $60 \mathrm{~km}$ over land (Donaldson, Gloster, Harvey, \& Deans, 1982; Donaldson \& Alexandersen, 2002; Kitching, 2002). Cloven hooved animals including livestock 
and wildlife species are mostly affected, with cases of the FMD virus having been isolated from cattle, sheep, goats, pigs, and some wildlife species (Anderson et al., 1979; Bengis, Thomson, Hedger, De Vos, \& Pini, 1986; Thompson, 1994; Vosloo, Bastos, Sangare, Hargreaves, \& Thomson, 2002; Ayebazibwe et al., 2010a; Miguel et al., 2013). Wildlife species such as buffalo, deer, gazelles, elands, Wilde beests, impalas, Harte beests, waterbucks and giraffes, and elephants have been cited to have a role in FMD epidemiology in eastern and southern Africa (Anderson et al., 1979; Vosloo et al., 2002; Vosloo, Bastos, Sahle, Sangare, \& Dwarka, 2005; Bengis, 2005; Ayebazibwe et al., 2010a; Miguel et al., 2013). As early as 1979, Anderson et al. (1979) documented the recovery of the FMDV from over $50 \%$ of oesophageal-pharyngeal samples from selected wild animals in Kenya. The wildlife species included buffalo, elands, gazelles, impala, giraffes, water bucks and wildebeests. Additionally, serological evidence of infection after exposure to the virus was detected from samples from buffalo, elands, gazelles, wildebeest and topi. Although buffalos have been implicated for spreading FMD because of their carrier status, no experimental evidence has been established to this effect (Anderson et al., 1979; Sutmoller \& Casas, 2002; Kitching et al., 2007). On the other hand, domesticated animals such as goats, sheep that hardly show clinical signs of FMD when infected, and have been implicated in some of the major global outbreaks that happened in the United Kingdom and Denmark in early 2000s (Sutmoller \& Casas, 2002; Kitching, 2002).

The World Health Organisation for Animal Health (OIE) has classified FMD to be among the most important notifiable diseases. Foot-and-mouth disease outbreaks require quick intervention because of the potentially rapid rate of disease spread and substantial negative economic impacts on livestock stakeholders (OIE, 2017). Losses such as reduced meat and milk yields, loss in draught power, control and treatment costs, and lack of or low sales during outbreaks are some of the negative impacts of FMD (Domingo et al., 1990; Domingo, Baranowski, \& Escarmis, 2002; James \& Rushton, 2002; Baluka, 2016; Casey-Bryars et al., 2018). Annual funds spent on vaccination in Africa, have been estimated at US \$ 830 million (James \& Rushton, 2002) while the total cost burden in Africa has been estimated at more than US \$ 6.5 billion per year (FAO, 2018). Countries that usually suffer from FMD outbreaks, have been shown to have low Gross Domestic Products (GDPs) compared to those where FMD has been eradicated (Jamal \& Belsham, 2013). The observation of such trends could be attributed to the high costs of controlling the disease and the incurred losses due to disease.

The FMDV exists as seven serotypes, namely; O, A, C, Asia 1 and Southern African Territories (SAT) 1-3 (Stanway, Brown, Christian, Hovi, \& Hypia, 2005; Jamal \& Belsham, 2013). These FMDV serotypes are grouped according to the nucleotide differences within the viral protein 1 (VP1) gene coding region (Jamal \& Belsham, 2013). This coding region has also been commonly used to infer antigenic diversity among the different serotypes (Knowles \& Samuel, 2003; Fry et al., 2005). The independent FMD virus serotypes are subdivided into topotypes which are further divided into lineages and sub-types still based on the VP1 sequence diversity (Jamal \& Belsham, 2013; WRLFMD). Globally, serotype O is the most predominant serotype and has been reported to have 11 geographic topotypes in circulation (Jamal \& Belsham, 2013). Within this serotype, topotypes East Africa (EA-1-4) have been found in circulation in eastern Africa, with EA-2 being the most prevailing topotype in Uganda and Tanzania (Knowles, Samuel, Davies, Midgley, \& Valarcher, 2005; Balinda et al., 2010a; Kasambula et al., 2012; Wekesa et al., 2013, Kasanga et al., 2015). Serotype A viruses have been clustered into three main topotypes, AFRICA, ASIA and EURO-SA which comprise over 26 different subtypes globally. The topotype that has been documented in circulation in East Africa is the AFRICA topotype and specifically genotypes I, II, IV and VII (Kasanga et al., 2015; Bari et al., 2014). The SAT serotypes have been predominantly found in Africa and were first isolated in South Africa. The SATs have been closely associated with wildlife-livestock interfaces and each SAT serotype has been isolated from the African buffalo in East African region (Vosloo et al., 2005; Ayebazibwe et al., 2010a; Kalema-Zikusoka, Bengis, Michel, \& Woodford, 2005; Wekesa et al., 2014). Serotype SAT 1 has 13 topotypes (I-XIII) and four of these have been in circulation in East Africa. These topotypes have been observed to have unique and distinct geographical distributions across the different countries in East Africa (Vosloo et al., 2002). The SAT 2 serotype comprised of fourteen documented topotypes (I-XIV) whereas SAT 3 has five topotypes (I-V). Serotype C has not been reported in circulation in East Africa since 2004 when it was last detected in Kenya (Sangula et al., 2010). The serotype is distributed into three topotypes EURO-SA, ASIA and AFRICA. Serotype Asia 1, is predominant in the Asian continent and has only one topotype in circulation, and has never been detected on the African continent (WRLFMD).

\subsection{Global Distribution of FMDV Serotypes}

The global distribution of FMDV has been influenced by factors such as: livestock density, grazing systems, animal movements, animal husbandry and livestock trade patterns, wildlife reservoirs and low capacities for 
disease control (Rweyamamu et al., 2008; Ayebazibwe et al., 2010b; Fèvre, Bronsvoort, Hamilton, \& Cleaveland, 2006; Namatovu et al., 2013). Although Europe has managed to control FMD, serotypes O, A, C and Asia 1 have been responsible for most of the incursions that have occurred (Valarcher et al., 2008). Four serotypes (O, A, C, Asia 1) have been documented in circulation on the Asian continent, with brief reported cases of SAT 1 and SAT 2 in the Middle East (Ahmed et al., 2012; Rweyemamu et al., 2008) while, South America has had three serotypes that have been reported in circulation (O, A, and C) (Valarcher et al., 2005). Six out of the seven existing FMDV serotypes have occurred in Africa (O, A, C, SAT 1, SAT 2, SAT 3) and particularly, all six of the seven serotypes have been detected in East Africa (Kivaria, 2003; Sangula et al., 2011; Kasanga et al., 2015, Casey-Bryars et al., 2018). This makes East Africa one of the regions which has had the highest diversity of FMDV serotypes which could consequently complicate FMD epidemiology and control. This is because FMDV vaccines do not offer cross protection (Kitching, 2002; Sahle, Dwarka, Venter, \& Vosloo, 2007). Generally, serotype $\mathrm{O}$ is most widespread globally, followed by serotype A. The SATs 1-3 are limited to Africa and are highly diverse. Although FMD is endemic in East African countries, serotype $\mathrm{C}$ has not been detected since 2004 (Sangula et al., 2011), may be because the serotype is in the process of becoming extinct or its detection has been overlooked. Serotype SAT 3 was previously isolated from the African Buffalo in 2007, but was last reported in 2015 having been isolated from a Ugandan Ankole calf (Dhikusooka et al., 2015).

Foot-and-mouth disease has been endemic both in Uganda and Tanzania since 1953 and 1927 respectively, with outbreaks reported more than once each year (Kivaria, 2003; Ayebazibwe et al., 2010b; Kasanga et al., 2012). Christensen et al. (2004) documented an increase in FMD cases in Uganda between 2000 and 2003. The same study reported 1 to 15 annual cases between 1996 and 1999; 1200 to 3100 annual cases between 2002 and 2003; 27000 cases in 2003 and an additional 18000 cases in the first six months of 2004. Between 2001 and 2008, 311 outbreaks were recorded in $70 \%$ of the districts in Uganda (Ayebazibwe et al., 2010b). In Tanzania, 878 outbreaks were reportedly occurred between 2001 and 2006, with variable numbers reported. The reports showed that most outbreaks occurred at locations close to international borders (Ayebazibwe et al., 2010b; Kivaria, 2003).

\subsection{Control of FMD in Uganda and Tanzania}

In most of the continents, mainly in sub-Saharan Africa, eradication of FMD may be considered as a long term objective (Kitching et al., 2007). Ideally, FMD control and eradiation in endemic settings should involve vaccination of livestock twice a year, control of livestock movement and assessment of the risks associated with introduction of FMD in disease free areas (Thomson et al., 2003; Bruckner et al., 2002; Jori et al., 2009, Maree et al., 2014). The use of FMD vaccines has been commonly used in Uganda to prevent spread of FMD outbreaks (Ministry of Livestock Animal Industry and Fisheries [MAAIF], 2009) with annual expenses of FMD vaccines estimated at US $\$ 58000$ to $\$ 1088820$ (Muleme et al., 2012). However, lack of routine vaccinations and reports of delayed and inadequate vaccination campaigns have been cited in causing disease spread to other areas (MAAIF, 2009). Issues with FMD laboratory diagnosis and poor reporting systems have increased the risk of disease spread and led to poor disease control options (MAAIF, 2009). In Tanzania, very low levels of vaccinations have been reported as most farmers do not vaccinate their animals (Railey, Lembo, Palmer, Shirima, \& Marsh, 2018; Hasler et al., 2017). The Animal Health Strategy and Vision for Tanzania emphasizes improved epidemiological surveillance, better response to outbreaks and improved laboratory diagnostic networks in order to eradicate FMD (Maziku, Mruttu, \& Gebru, 2016). The trivalent FMD vaccine used then in Uganda contained strains for serotypes O, SAT1 and SAT 2 (Namatovu et al., 2015), while in Tanzania the multivalent vaccine commonly used consists of serotype O, A, SAT-1 and SAT-2 (Balinda et al., 2010a; Sallu et al., 2015). Vaccination against FMD especially in endemic areas remains complex because of multiplicity of antigenic types and subtypes given that there is limited cross protection between serotypes or topotypes within the given serotypes (Kitching et al., 2007). Issues such as poor knowledge of circulating strains, vaccine expense and the short time of protection elicited by the vaccine (often as little as 6 months) hinder the success of vaccination schemes in endemic countries (Kitching et al., 2007; Maree et al., 2014). Although the restriction of livestock and product movement is a control strategy emphasized by both countries, especially in Uganda (Kasambula et al., 2012), it still lacks proper enforcement (East African Community [EAC], 2004; Balinda et al., 2010b), making disease control difficult.

\subsubsection{Progressive Control Pathway-FMD}

The progressive control pathway for FMD was introduced by the FAO/OIE/EU-FMD to enable countries that are still endemic with FMD to develop strategies centred around existing information on the FMD status in the countries. The initial stages of the pathway, in which Uganda and Tanzania are in, are critical because comprehensive information on FMD is collected so as to move from stage 0 to 1 (FAO, 2011; FAO, 2018). The 
pathway has five steps through which a region/country should go until they reach a status of free without vaccination. For countries in stages 1-4 vaccination is one of the major control strategies and thus basic information on serotypes and even topotypes in circulation in that region are important (FAO, 2011).

With the recent start of the PCP-FMD implementation in East Africa in 2012, one of the vital requirements in the initial stages is the need for better understanding and knowledge on FMD epidemiology in a given country/region. Since Uganda and Tanzania are still in the initial stages of the PCP-FMD, where vaccination plays an important role in FMD control, this review aims to provide important information that can be used to develop effective vaccination strategies based on the spatial and temporal distribution of FMD serotypes in both countries between 2003 and 2013.

\section{Review Methodology}

\subsection{Data Base Search}

The methodology used was adapted from Khan, Regina Kunz, Kleijnen, and Antes (2003). Relevant questions were framed for the review, after which relevant work was identified, the quality of the selected studies was assessed, evidence from the reviewed articles summarised and finally information from the compiled evidence interpreted.

The PubMed Central, Google scholar, Science Direct databases were searched using key words "foot-and-mouth disease" OR "foot and mouth disease" AND "Tanzania" and "foot-and-mouth disease" OR "foot and mouth disease" AND "Uganda". The search included theses, books, conference proceedings as well as project reports. A total of 59 items were found relevant to the topic, the years (2003-2015) and the defined geographical study area. Abstracts were exported to Mendeley and were later viewed to determine if they met the selection criteria on whether to be included in the study or not. Additionally, Government reports and other relevant documents were retrieved in both hard and soft copy.

\subsection{Selection Criteria}

Studies in which analysed samples were collected before 2003 and after 2015 were not included in this paper. This was done in order to have a more comprehensive picture of what had transpired in eleven years before the PCP-FMD was implemented in East Africa and shortly after its implementation. The review only considered studies where FMD diagnosis was either performed using cell culture, loop-mediated isothermal amplification (LAMP), antigen-enzyme Linked Immuno-sorbent Assays (Ag-ELISA) or polymerase Chain reaction (PCR). This is because the above mentioned laboratory methods are able to detect for the presence of FMDV in an appropriate sample and are serotype specific (OIE, 2012). The criteria included articles on buffalo but excluded articles on other wild animals. It also included articles on other livestock species including cattle. In the end, a total of 39 articles were selected for consideration.

\subsection{Data Retrieval}

Articles whose abstracts had been included for the study were then retrieved in full text format and reviewed by the first author as to whether they met the inclusion criteria. All articles reviewed in the study were in the English language. Qualitative data were compiled on country, year, serotypes, topotype, accession number, location from which samples were collected and referenced (Table 1).

Table 1. Foot-and-mouth disease viruses used in study

\begin{tabular}{|c|c|c|c|c|c|c|c|c|}
\hline $\mathbf{S} / \mathbf{N}$ & Country & Year & Virus name & Serotype & $\begin{array}{l}\text { Topotype/ } \\
\text { Lineage }\end{array}$ & $\begin{array}{l}\text { Accession } \\
\text { Number }\end{array}$ & Location & Reference \\
\hline 1 & Uganda & 2003 & $\mathrm{O} / \mathrm{UGA} / 7 / 03$ & $\mathrm{O}$ & $\mathrm{NM}$ & EU919243 & Unknown & $\begin{array}{l}\text { Chitray, de Beer, } \\
\text { Vosloo, \& Maree, } 2014\end{array}$ \\
\hline 2 & Uganda & 2003 & UGA/5/03/Masaka & $\mathrm{O}$ & NM & AY349955 & Masaka, Central region & Sahle, 2004 \\
\hline 3 & Uganda & 2003 & UGA/7/03/Hoima & $\mathrm{O}$ & NM & AY349956 & Hoima, Western region & Sahle, 2004 \\
\hline 4 & Uganda & 2003 & UGA/4/03/Jinja & $\mathrm{O}$ & NM & AY349954 & Jinja, Eastern region & Sahle, 2004 \\
\hline 5 & Uganda & 2003 & UGA/3/03Jinja & $\mathrm{O}$ & NM & AY349953 & Jinja, Eastern region & Sahle, 2004 \\
\hline 6 & Uganda & 2004 & $\mathrm{U} 20 \mathrm{~B} / 04$ & $\mathrm{O}$ & EA-2 & HM756621 & Hoima, Western region & Balinda et al., 2010a \\
\hline 7 & Uganda & 2004 & $\mathrm{U} 17 \mathrm{~B} / 04$ & $\mathrm{O}$ & EA-2 & HM756620 & Hoima, Western region & Balinda et al., 2010a \\
\hline 8 & Uganda & 2004 & $\mathrm{U} 14 \mathrm{~B} / 04$ & $\mathrm{O}$ & EA-2 & HM756619 & Hoima, Western region & Balinda et al., 2010a \\
\hline 9 & Uganda & 2004 & $\mathrm{U} 13 \mathrm{~B} / 04$ & $\mathrm{O}$ & EA-2 & HM756618 & Hoima, Western region & Balinda et al., 2010a \\
\hline 10 & Uganda & 2004 & UGA/12/2004 & SAT 2 & I & GU323179 & Kiboga, Central region & Namatovu et al., 2013 \\
\hline 11 & Uganda & 2004 & UGA/11/2004 & SAT 2 & I & GU323178 & Kiboga, Central region & Balinda et al., 2010b \\
\hline 12 & Uganda & 2004 & UGA/09/2004 & SAT 2 & I & GU323177 & Kiboga, Central region & Balinda et al., 2010b \\
\hline
\end{tabular}




\begin{tabular}{|c|c|c|c|c|c|c|c|c|}
\hline 13 & Uganda & 2004 & UGA/08/2004 & SAT 2 & I & GU323176 & Kiboga, Central region & Balinda et al., 2010b \\
\hline 14 & Uganda & 2004 & UGA/07/2004 & SAT 2 & I & GU323175 & Kiboga, Central region & Balinda et al., 2010b \\
\hline 15 & Uganda & 2004 & UGA/05/2004 & SAT 2 & I & GU323174 & Kiboga, Central region & Balinda et al., 2010b \\
\hline 16 & Uganda & 2004 & UGA/03/2004 & SAT 2 & I & GU323173 & Kiboga, Central region & Balinda et al., 2010b \\
\hline 17 & Uganda & 2004 & UGA/02/2004 & SAT 2 & I & GU323172 & Kiboga, Central region & Balinda et al., 2010b \\
\hline 18 & Uganda & 2004 & UGA/01/2004 & SAT 2 & I & GU323171 & Kiboga, Central region & Balinda et al., 2010b \\
\hline 19 & Uganda & 2005 & $\mathrm{U} 12 / 05$ & $\mathrm{O}$ & EA-2 & HM756625 & Wakiso, Central region & Balinda et al., 2010a \\
\hline 20 & Uganda & 2006 & $\mathrm{U} 25 / 06$ & $\mathrm{O}$ & EA-2 & HM756628 & Mpigi, Central region & Balinda et al., 2010a \\
\hline 21 & Uganda & 2006 & U18/06 & $\mathrm{O}$ & EA-2 & HM756627 & Mpigi, Central region & Balinda et al., 2010a \\
\hline 22 & Uganda & 2006 & $\mathrm{O} / \mathrm{U} / 25 / 2006$ & $\mathrm{O}$ & EA-2 & HM625677 & Mpigi, Central region & Balinda et al., 2010a \\
\hline 23 & Uganda & 2006 & $\mathrm{O} / \mathrm{U} / 312 / 2006$ & $\mathrm{O}$ & EA-2 & HM191257 & Mbarara, Western region & Balinda et al., 2010a \\
\hline 23 & Uganda & 2006 & O/Uganda/2006 & $\mathrm{O}$ & EA-2 & EF611987 & - & Klein et al., Unpublished \\
\hline 24 & Uganda & 2008 & OUGA2008KAMULI & $\mathrm{O}$ & EA-2 & JN974312 & Kamuli, Eastern region & Kasambula et al., 2012 \\
\hline 25 & Uganda & 2009 & OUGA2009AMOLATAR & $\mathrm{O}$ & EA-2 & JN974311 & Amolatar, Northern region & Kasambula et al., 2012 \\
\hline 26 & Uganda & 2009 & OUGA2009DOKOLO & $\mathrm{O}$ & EA-2 & JN974310 & Dokolo, Northern region & Kasambula et al., 2012 \\
\hline 27 & Uganda & 2009 & OUGA2009LIRA & $\mathrm{O}$ & EA-2 & JN974308 & Lira, Northern region & Kasambula et al., 2012 \\
\hline 28 & Uganda & 2009 & OUGA2009APAC & $\mathrm{O}$ & EA-2 & JN974309 & Apac, Northern region & Kasambula et al., 2012 \\
\hline 29 & Uganda & 2010 & 18RAKAI & $\mathrm{O}$ & EA-2 & KC987540 & Rakai, Central Uganda & Kerfua et al., 2013 \\
\hline 30 & Uganda & 2011 & $\mathrm{U} 24 / 11$ & $\mathrm{O}$ & EA-2 & KF478941 & Sembabule, Central region & Namatovu et al., 2015 \\
\hline 31 & Uganda & 2011 & $\mathrm{U} 19 / 11$ & $\mathrm{O}$ & EA-2 & KF478940 & Kumi, Eastern region & Namatovu et al., 2015 \\
\hline 32 & Uganda & 2011 & U07/11 & $\mathrm{O}$ & EA-2 & KF478939 & Rakai. Western region & Namatovu et al., 2015 \\
\hline 33 & Uganda & 2011 & U04/11 & $\mathrm{O}$ & EA-2 & KF478938 & Bukedea, Central region & Namatovu et al., 2015 \\
\hline 34 & Uganda & 2011 & UGA-161-13 & SAT 1 & IV (EA-1) & KP025679 & Kasese, Western region & Dhikusooka et al., 2016 \\
\hline 35 & Uganda & 2011 & UGA-116-13 & SAT 1 & IV (EA-1) & KP025678 & Kasese, Western region & Dhikusooka et al., 2016 \\
\hline 36 & Uganda & 2013 & $\mathrm{U} 75 / 13$ & A & AFRICA/G-I & KP089985 & Wakiso, Central region & Namatovu et al., 2015 \\
\hline 37 & Uganda & 2013 & $\mathrm{U} 74 / 13$ & A & AFRICA/G-I & KP089984 & Wakiso, Central region & Namatovu et al., 2015 \\
\hline 38 & Uganda & 2013 & $\mathrm{U} 73 / 13$ & SAT 2 & I & KP089990 & Isingiro, Western region & Namatovu et al., 2015 \\
\hline 39 & Uganda & 2013 & $\mathrm{U} 45 / 13$ & SAT 2 & I & KP089988 & Isingiro, Western region & Namatovu et al., 2015 \\
\hline 40 & Uganda & 2013 & $\mathrm{U} 69 / 13$ & SAT 2 & I & KР089989 & Isingiro, Western region & Namatovu et al., 2015 \\
\hline 41 & Uganda & 2013 & $\mathrm{U} 30 / 13$ & SAT 2 & I & KP089986 & Isingiro, Western region & Namatovu et al., 2015 \\
\hline 42 & Uganda & 2013 & $\mathrm{U} 35 / 13$ & SAT 2 & I & KP089987 & Isingiro, Western region & Namatovu et al., 2015 \\
\hline 43 & Uganda & 2013 & $\mathrm{UGA} / 1 / 13$ & SAT 3 & IV & KJ820999 & Kasese, Western region & Dhikusooka et al., 2015 \\
\hline 44 & Uganda & 2014 & O/BKD-009-P & $\mathrm{O}$ & NM & KY548429 & Bukedea, Eastern region & Genbank \\
\hline 45 & Uganda & 2014 & $\mathrm{O} / \mathrm{BKD} / 016-\mathrm{P}$ & $\mathrm{O}$ & NM & KY548426 & Bukedea, Eastern region & Genbank \\
\hline 46 & Uganda & 2014 & O/ALB-F4-060-P & $\mathrm{O}$ & NM & KY548412 & Alebtong, Northern region & Genbank \\
\hline 47 & Uganda & 2014 & O/NKP-79-P & $\mathrm{O}$ & NM & KY558315 & Nakapiripirit, Eastern region & Genbank \\
\hline 48 & Uganda & 2014 & O/MAN-014-P & $\mathrm{O}$ & NM & KY558311 & Manafwa, Eastern region & Genbank \\
\hline 49 & Uganda & 2014 & SAT 2/KIR-007-P & SAT 2 & NM & KY558291 & Kirihura, Western region & Genbank \\
\hline 50 & Uganda & 2014 & SAT 2/KIR-91-P & SAT 2 & NM & KY558289.1 & Kirihura, Western region & Genbank \\
\hline 51 & Uganda & 2014 & O/MBE-3-T-2014 & $\mathrm{O}$ & NM & KY558284.1 & Mbale, Eastern region & Genbank \\
\hline 52 & Uganda & 2014 & O/KUM 27-P-2014 & $\mathrm{O}$ & NM & KY548478.1 & Kumi, Eastern region & Genbank \\
\hline 53 & Uganda & 2014 & O/KAB-394-P & $\mathrm{O}$ & NM & KY548473.1 & Kaabong, Eastern region & Genbank \\
\hline 54 & Uganda & 2015 & O/BUG-89-P & $\mathrm{O}$ & NM & KY548440.1 & Bugiri, Eastern region & Genbank \\
\hline 55 & Uganda & 2015 & O/BUG-82-P & $\mathrm{O}$ & NM & KY548438 & Bugiri, Eastern region & Genbank \\
\hline 56 & Uganda & 2015 & O/BUG-77-P & $\mathrm{O}$ & NM & KY548438 & Bugiri, Eastern region & Genbank \\
\hline 57 & Uganda & 2015 & O/BUG-69-P & $\mathrm{O}$ & NM & KY548435.1 & Bugiri, Eastern region & Genbank \\
\hline 58 & Uganda & 2015 & $\mathrm{O} / \mathrm{NGO}-372-\mathrm{P}$ & $\mathrm{O}$ & NM & KY558336.1 & Ngora, Eastern region & Genbank \\
\hline 59 & Uganda & 2015 & O/NAP-189-P & $\mathrm{O}$ & NM & KY558335.1 & Napak, Eastern region & Genbank \\
\hline 60 & Uganda & 2015 & O/KYA-105-SW & $\mathrm{O}$ & NM & KY558333.1 & Kyankwanzi, Central region & Genbank \\
\hline 61 & Uganda & 2015 & O/SER-298-P & $\mathrm{O}$ & NM & KY558322.1 & Serere, Eastern region & Genbank \\
\hline 62 & Uganda & 2015 & O/KAY-90-P & $\mathrm{O}$ & NM & KY548474.1 & Kiryandongo, Central region & Genbank \\
\hline 63 & Uganda & 2015 & O/BUS-406-P & $\mathrm{O}$ & NM & KY548461.1 & Busia, Eastern region & Genbank \\
\hline 64 & Uganda & 2015 & SAT 1/KIR-208-P & SAT 1 & NM & KY558307.1 & Kirihura, Western region & Genbank \\
\hline 65 & Tanzania & 2004 & TAN/18/2004 & SAT 2 & IV & KF561725 & Nkasi, Rukwa & Kasanga et al., 2012 \\
\hline 66 & Tanzania & 2004 & TAN/21/2004 & SAT 2 & IV & KF561726 & Morogoro & Kasanga et al., 2012 \\
\hline 67 & Tanzania & 2004 & TAN/9/2004 & SAT 2 & IV & KF561724 & Arusha & Kasanga et al., 2012 \\
\hline 68 & Tanzania & 2004 & TAN/6/2004 & SAT 2 & IV & KF561723 & Arusha & Kasanga et al., 2012 \\
\hline 69 & Tanzania & 2004 & TAN/4/2004 & SAT 2 & IV & KF561722 & Arusha & Kasanga et al., 2012 \\
\hline 70 & Tanzania & 2004 & TAN/17/2004 & $\mathrm{O}$ & EA-2 & KF561683 & Nkasi, Rukwa & Kasanga et al., 2012 \\
\hline 71 & Tanzania & 2004 & TAN/14/2004 & $\mathrm{O}$ & EA-2 & KF561682 & Ruvuma & Kasanga et al., 2012 \\
\hline 72 & Tanzania & 2004 & TAN/12/2004 & $\mathrm{O}$ & EA-2 & KF561681 & Chunya, Mbeya & Kasanga et al., 2012 \\
\hline 73 & Tanzania & 2004 & TAN/3/2004 & $\mathrm{O}$ & EA-2 & KF561680 & Iringa & Kasanga et al., 2012 \\
\hline
\end{tabular}




\begin{tabular}{|c|c|c|c|c|c|c|c|c|}
\hline 74 & Tanzania & 2004 & $\mathrm{TAN} / 2 / 2004$ & $\mathrm{O}$ & EA-2 & KF561679 & Kibaha, Pwani & Kasanga et al., 2012 \\
\hline 75 & Tanzania & 2004 & TAN/1/2004 & $\mathrm{O}$ & EA-2 & KF561678 & Bagamoyo & Kasanga et al., 2012 \\
\hline 76 & Tanzania & 2004 & TAN/2/2004 & $\mathrm{O}$ & EA-2 & KJ831706 & NM & Reeve et al., 2016 \\
\hline 77 & Tanzania & 2008 & TAN/11/2008 & A & AFRICA/G-I & KF561690 & Iringa & Kasanga et al., 2012 \\
\hline 78 & Tanzania & 2008 & TAN/12/2008 & A & AFRICA/G-I & KF561691 & Iringa & Kasanga et al., 2012 \\
\hline 79 & Tanzania & 2008 & TAN/16/2008 & $\mathrm{O}$ & EA-2 & KF561684 & Morogoro & Kasanga et al., 2012 \\
\hline 80 & Tanzania & 2008 & A/TAN-CVL-155 & A & AFRICA/ G-I & KJ947815 & Rukwa & Sallu et al., 2015 \\
\hline 81 & Tanzania & 2009 & A/TAN-CVL-042 & A & AFRICA/ G-I & KJ947814 & Dar es salaam & Sallu et al., 2015 \\
\hline 82 & Tanzania & 2009 & A/TAN-CVL-052 & A & AFRICA/G-I & KJ947812 & Dar es salaam & Sallu et al., 2015 \\
\hline 83 & Tanzania & 2009 & A/TAN-CVL-052b & A & AFRICA/G-I & KJ947822 & Dar es salaam & Sallu et al., 2015 \\
\hline 84 & Tanzania & 2009 & TAN/47/2009 & A & AFRICA/G-I & KF561697 & Bagamoyo & Kasanga et al., 2012 \\
\hline 85 & Tanzania & 2009 & TAN/43/2009 & SAT 2 & I & KF561727 & Makete & Kasanga et al., 2012 \\
\hline 86 & Tanzania & 2009 & TAN/45/2009 & A & AFRICA/G-I & KF561696 & Iringa & Kasanga et al., 2012 \\
\hline 87 & Tanzania & 2009 & TAN/42/2009 & A & AFRICA/G-1 & KF561695 & ,Dodoma & Kasanga et al., 2012 \\
\hline 88 & Tanzania & 2009 & TAN/11/2009 & $\mathrm{A}$ & AFRICA/G-1 & KF561694 & Kibaha, Pwani & Kasanga et al., 2012 \\
\hline 89 & Tanzania & 2009 & TAN/9/2009 & A & AFRICA/G-1 & KF561693 & Njombe & Kasanga et al., 2012 \\
\hline 90 & Tanzania & 2009 & TAN/4/2009 & A & AFRICA/G-1 & KF561692 & Morogoro & Kasanga et al., 2012 \\
\hline 91 & Tanzania & 2009 & TAN/44/2009 & $\mathrm{O}$ & EA-2 & KF561686 & Njombe & Kasanga et al., 2012 \\
\hline 92 & Tanzania & 2009 & TAN/5/2009 & $\mathrm{O}$ & EA-2 & KF561685 & Morogoro & Kasanga et al., 2012 \\
\hline 93 & Tanzania & 2009 & O/TAN/5/2009 & $\mathrm{O}$ & NM & KR149722 & $?$ & $\mathrm{Xu}$ et al., Unpublished \\
\hline 94 & Tanzania & 2009 & O/TAN-CVL-040 & $\mathrm{O}$ & EA-2 & KJ947805 & Iringa & Sallu et al., 2015 \\
\hline 95 & Tanzania & 2010 & N/A & SAT $2 *$ & N/A & NA & $?$ & $\begin{array}{l}\text { Mwanandota, Kasanga, } \\
\text { Yongolo, Sallu, } \\
\text { \& Mkama, } 2013\end{array}$ \\
\hline 96 & Tanzania & 2010 & $\mathrm{~N} / \mathrm{A}$ & $\mathrm{O}^{*}$ & N/A & NA & $?$ & Mwandandota et al., 2013 \\
\hline 97 & Tanzania & 2010 & O/TAN-CVL-039 & $\mathrm{O}$ & EA-2 & KJ947823 & Mwanza & Sallu et al., 2015 \\
\hline 98 & Tanzania & 2010 & O/TAN-CVL-0144 & $\mathrm{O}$ & EA-2 & KJ947831 & Mwanza & Sallu et al., 2015 \\
\hline 99 & Tanzania & 2010 & O/TAN-CVL-0142 & $\mathrm{O}$ & EA-2 & KJ947824 & Mwanza & Sallu et al., 2015 \\
\hline 100 & Tanzania & 2010 & O/TAN-CVL-008 & $\mathrm{O}$ & EA-2 & KJ947810 & Mwanza & Sallu et al,2015 \\
\hline 101 & Tanzania & 2010 & O/TAN-CVL-009 & $\mathrm{O}$ & EA-2 & KJ947807 & Mwanza & Sallu et al., 2015 \\
\hline 102 & Tanzania & 2010 & O/TAN-CVL-086 & $\mathrm{O}$ & EA-2 & KJ947832 & Shinyanga & Sallu et al., 2015 \\
\hline 103 & Tanzania & 2010 & O/TAN-CVL-011 & $\mathrm{O}$ & EA-2 & KJ947808 & Mara & Sallu et al., 2015 \\
\hline 104 & Tanzania & 2010 & O/TAN-CVL-037 & $\mathrm{O}$ & EA-2 & KJ947806 & Tabora & Sallu et al., 2015 \\
\hline 105 & Tanzania & 2010 & O/TAN-CVL-046 & $\mathrm{O}$ & EA-2 & KJ947827 & Tabora & Sallu et al. 2015 \\
\hline 106 & Tanzania & 2011 & O/TAN-CVL-015 & $\mathrm{O}$ & EA-2 & KJ947828 & Mara & Sallu et al., 2015 \\
\hline 107 & Tanzania & 2010 & O/TAN-CVL-006 & $\mathrm{O}$ & EA-2 & KJ947830 & Mara & Sallu et al., 2015 \\
\hline 108 & Tanzania & 2010 & O/TAN-CVL-013 & $\mathrm{O}$ & EA-2 & KJ947829 & Mara & Sallu et al., 2015 \\
\hline 109 & Tanzania & 2010 & O/TAN-CVL-014 & $\mathrm{O}$ & EA-2 & KJ947833 & Mara & Sallu et al., 2015 \\
\hline 110 & Tanzania & 2010 & O/TAN-CVL-139 & $\mathrm{O}$ & EA-2 & KJ947825 & Mara & Sallu et al., 2015 \\
\hline 111 & Tanzania & 2010 & O/TAN-CVL-040 & $\mathrm{O}$ & EA-2 & KJ947805 & Iringa & Sallu et al 2015 \\
\hline 112 & Tanzania & 2010 & O/TAN-CVL-019 & $\mathrm{O}$ & EA-2 & KJ947811 & Rukwa & Sallu et al., 2015 \\
\hline 113 & Tanzania & 2010 & O/TAN-CVL-109 & $\mathrm{O}$ & EA-2 & KJ947836 & Dar Es Salaam & Sallu et al., 2015 \\
\hline 114 & Tanzania & 2010 & O/TAN-CVL-004 & $\mathrm{O}$ & EA-2 & KJ947826 & Kagera & Sallu et al., 2015 \\
\hline 115 & Tanzania & 2010 & O/TAN-CVL-108 & $\mathrm{O}$ & EA-2 & N/A & Mtwara & Sallu et al., 2015 \\
\hline 116 & Tanzania & 2010 & O/TAN-CVL-0116 & $\mathrm{O}$ & EA-2 & N/A & Zanzibar & Sallu et al., 2015 \\
\hline 117 & Tanzania & 2010 & O/TAN-CVL-071 & $\mathrm{O}$ & EA-2 & $\mathrm{N} / \mathrm{A}$ & Morogoro & Sallu et al., 2015 \\
\hline 118 & Tanzania & 2010 & O/TAN-CVL-017 & $\mathrm{O}$ & EA-2 & N/A & Rukwa & Sallu et al., 2015 \\
\hline 119 & Tanzania & 2010 & O/TAN-CVL-034 & $\mathrm{O}$ & EA-2 & N/A & Tabora & Sallu et al., 2015 \\
\hline 120 & Tanzania & 2010 & O/TAN-CVL-047 & $\mathrm{O}$ & EA-2 & N/A & Tabora & Sallu et al., 2015 \\
\hline 121 & Tanzania & 2011 & KJ947819 & A & AFRICA/G-I & - & Kigoma & Sallu et al., 2015 \\
\hline 122 & Tanzania & 2011 & KJ947813 & A & AFRICA/G-I & - & Tabora & Sallu et al,2015 \\
\hline 123 & Tanzania & 2011 & KJ947821 & A & AFRICA/G-I & - & Mtwara & Sallu et al., 2015 \\
\hline 124 & Tanzania & 2011 & KJ947837 & A & AFRICA/G-I & - & Dar es salaam & Sallu et al., 2015 \\
\hline 125 & Tanzania & 2011 & A/TAN/CVL-160 & A & AFRICA/G-I & - & Dar es salaam & Sallu et al., 2015 \\
\hline 126 & Tanzania & 2011 & A/TAN/CVL-289 & $\mathrm{A}$ & AFRICA/G-I & - & Kigoma & Sallu et al., 2015 \\
\hline 127 & Tanzania & 2011 & TAN/CVL-036 & $\mathrm{O}$ & EA-2 & N/A & Tabora & Sallu et al., 2015 \\
\hline 128 & Tanzania & 2011 & TAN/CVL-109 & $\mathrm{O}$ & EA-2 & N/A & Dar es salaam & Sallu et al., 2015 \\
\hline 129 & Tanzania & 2011 & TAN/CVL-031 & $\mathrm{O}$ & EA-2 & N/A & Coastal region & Sallu et al., 2015 \\
\hline 130 & Tanzania & 2011 & TAN/CVL-039 & $\mathrm{O}$ & EA-2 & N/A & Mwanza & Sallu et al., 2015 \\
\hline 131 & Tanzania & 2011 & TAN/6/2011 & SAT 2 & NM & MF592599.1 & Arusha & Casey-Bryars et al., 2018 \\
\hline 132 & Tanzania & 2011 & O/TAN-CVL-015 & $\mathrm{O}$ & EA-2 & KJ947828 & Mara & Sallu et al., 2015 \\
\hline 133 & Tanzania & 2011 & TAN/4/2011 & SAT2 & NM & MF592598.1 & Arusha & Casey-Bryars et al., 2018 \\
\hline
\end{tabular}




\begin{tabular}{|c|c|c|c|c|c|c|c|c|}
\hline 134 & Tanzania & 2011 & TAN/8/2011 & SAT 2 & NM & MF592601 & Manyara & Casey-Bryars et al., 2018 \\
\hline 135 & Tanzania & 2012 & TAN/5/2012 & SAT 2 & NM & KM268900 & Serengeti & Logan et al., 2014 \\
\hline 136 & Tanzania & 2012 & TAN/22/2012 & SAT $1 *$ & NM & KM268899 & Simanjiro & Logan et al., 2014 \\
\hline 137 & Tanzania & 2012 & O/TAN-CVL-321 & $\mathrm{O}$ & EA-2 & KJ947835 & Mbeya & Sallu et al., 2017 \\
\hline 138 & Tanzania & 2012 & O/TAN-CVL-318 & $\mathrm{O}$ & EA-2 & KJ947834 & Mbeya & Sallu et al., 2015 \\
\hline 139 & Tanzania & 2012 & TAN/37/2012 & SAT 2 & $\mathrm{NM}$ & MF592622.1 & Serengeti, Mara, & Casey-Bryars et al., 2018 \\
\hline 140 & Tanzania & 2012 & TAN/25/2012 & SAT 1 & NM & MF592618 & Simanjiro, Mara & Casey-Bryars et al., 2018 \\
\hline 141 & Tanzania & 2012 & TAN/19/2012 & SAT 2 & NM & MF592615 & Simanjiro, Mara & Casey-Bryars et al., 2018 \\
\hline 142 & Tanzania & 2012 & TAN/3/2012 & SAT 2 & $\mathrm{NM}$ & MF592603 & Bunda, Mara & Casey-Bryars et al., 2018 \\
\hline 143 & Tanzania & 2012 & TAN/73/2012 & A & NM & MF592643 & Serengeti, Mara & Casey-Bryars et al., 2018 \\
\hline 144 & Tanzania & 2012 & TAN/45/2012 & SAT 1 & NM & MF592628 & Manyara & Casey-Bryars et al., 2018 \\
\hline 145 & Tanzania & 2012 & TAN/41/2012 & A & $\mathrm{NM}$ & MF592626 & Arusha & Casey-Bryars et al., 2018 \\
\hline 146 & Tanzania & 2012 & TAN/39/2012 & $\mathrm{O}$ & $\mathrm{NM}$ & MF592624 & Arusha & Casey-Bryars et al., 2018 \\
\hline 147 & Tanzania & 2012 & TAN/27/2012 & SAT 1 & NM & MF592644.1 & Serengeti, Mara & Casey-Bryars et al., 2018 \\
\hline 148 & Tanzania & 2012 & SAT1/TAN-CVL-355 & SAT 1 & I (NWZ) & - & Morogoro & Sallu et al., 2015 \\
\hline 149 & Tanzania & 2012 & SAT1/TAN-CVL-359 & SAT 1 & I (NWZ) & - & Dar es salaam & Sallu et al., 2015 \\
\hline 150 & Tanzania & 2012 & SAT1/TAN-CVL-360 & SAT 1 & I (NWZ) & - & Dar es salaam & Sallu et al., 2015 \\
\hline 151 & Tanzania & 2012 & SAT1/TAN-CVL-361 & SAT 1 & I (NWZ) & - & Dar es salaam & Sallu et al., 2015 \\
\hline 152 & Tanzania & 2012 & O/TAN/CVL-109 & $\mathrm{O}$ & EA-2 & - & Dar es salaam & Sallu et al., 2015 \\
\hline 153 & Tanzania & 2013 & $\mathrm{TAN} / 27 / 2013$ & A & NM & MF592660 & Serengeti, Mara & Casey-Bryars et al., 2018 \\
\hline 154 & Tanzania & 2013 & TAN/30/2013 & SAT 1 & NM & MF592662 & Serengeti, Mara & Casey-Bryars et al., 2018 \\
\hline 155 & Tanzania & 2013 & O/TAN-CVL-452 & $\mathrm{O}$ & EA-2 & N/A & Lindi & Sallu et al., 2015 \\
\hline 156 & Tanzania & 2013 & O/TAN/CVL-364 & $\mathrm{O}$ & EA-2 & N/A & Iringa & Sallu et al., 2015 \\
\hline 157 & Tanzania & 2013 & O/TAN/CVL-366 & $\mathrm{O}$ & EA-2 & N/A & Iringa & Sallu et al., 2015 \\
\hline 158 & Tanzania & 2013 & O/TAN/CVL-376 & $\mathrm{O}$ & EA-2 & N/A & Kagera & Sallu et al., 2015 \\
\hline 159 & Tanzania & 2013 & O/TAN/CVL-378 & $\mathrm{O}$ & EA-2 & N/A & Kagera & Sallu et al., 2015 \\
\hline 160 & Tanzania & 2013 & $\mathrm{O} / / \mathrm{TAN}-\mathrm{CVL}-362$ & $\mathrm{O}$ & EA-2 & N/A & Iringa & Sallu et al., 2015 \\
\hline 161 & Tanzania & 2013 & A/TAN-CVL-293 & A & AFRICA/G-1 & - & Kigoma & Sallu et al., 2015 \\
\hline 162 & Tanzania & 2013 & A/TAN-CVL-370 & $\mathrm{A}$ & AFRICA/G-1 & - & Kagera & Sallu et al., 2015 \\
\hline 163 & Tanzania & 2013 & A/TAN-CVL-374 & A & AFRICA/G-1 & - & Kagera & Sallu et al., 2015 \\
\hline 164 & Tanzania & 2013 & SAT1/TAN-CVL-354 & SAT 1 & I (NWZ) & - & Morogoro & Sallu et al., 2015 \\
\hline 165 & Tanzania & 2014 & TAN/38/2014 & SAT 1 & NM & MF592695.1 & Serengeti, Mara & Casey-Bryars et al., 2018 \\
\hline 166 & Tanzania & 2014 & TAN/41/2014 & SAT 1 & NM & MF592696 & Serengeti, Mara & Casey-Bryars et al., 2018 \\
\hline 167 & Tanzania & \# & N/A & SAT $2 *$ & N/A & N/A & Kagera & Kasanga et al., 2012 \\
\hline 168 & Tanzania & \# & N/A & SAT $2 *$ & N/A & N/A & Arusha & Kasanga et al., 2012 \\
\hline 169 & Tanzania & \# & N/A & SAT $2 *$ & N/A & N/A & Kilimanjaro & Kasanga et al., 2012 \\
\hline 170 & Tanzania & \# & N/A & SAT $2 *$ & N/A & N/A & Pwani & Kasanga et al., 2012 \\
\hline 171 & Tanzania & \# & N/A & SAT $2 *$ & $\mathrm{~N} / \mathrm{A}$ & N/A & Tabora & Kasanga et al., 2012 \\
\hline 172 & Tanzania & \# & N/A & SAT $2 *$ & N/A & N/A & Rukwa & Kasanga et al., 2012 \\
\hline 173 & Tanzania & \# & N/A & SAT $2 *$ & $\mathrm{~N} / \mathrm{A}$ & N/A & Mbeya & Kasanga et al., 2012 \\
\hline 174 & Tanzania & \# & N/A & SAT $2 *$ & N/A & N/A & Iringa & Kasanga et al., 2012 \\
\hline 175 & Tanzania & \# & N/A & SAT $2 *$ & N/A & N/A & Morogoro & Kasanga et al., 2012 \\
\hline 176 & Tanzania & \# & N/A & SAT $2 *$ & N/A & N/A & Singida & Kasanga et al., 2012 \\
\hline 177 & Tanzania & \# & N/A & SAT $2 *$ & $\mathrm{~N} / \mathrm{A}$ & N/A & Kigoma & Kasanga et al., 2012 \\
\hline 178 & Tanzania & \# & $\mathrm{N} / \mathrm{A}$ & SAT $1 *$ & $\mathrm{~N} / \mathrm{A}$ & N/A & Dar es salaam & Kasanga et al., 2012 \\
\hline 179 & Tanzania & \# & N/A & $\mathrm{O}^{*}$ & $\mathrm{~N} / \mathrm{A}$ & N/A & Dar es salaam & Kasanga et al., 2012 \\
\hline 180 & Tanzania & \# & N/A & $\mathrm{O}^{*}$ & $\mathrm{~N} / \mathrm{A}$ & $\mathrm{N} / \mathrm{A}$ & Arusha & Kasanga et al., 2012 \\
\hline 181 & Tanzania & \# & N/A & $\mathrm{O}^{*}$ & N/A & N/A & Kagera & Kasanga et al., 2012 \\
\hline 182 & Tanzania & \# & N/A & $\mathrm{O}^{*}$ & $\mathrm{~N} / \mathrm{A}$ & NM & Mbeya & Kasanga et al., 2012 \\
\hline 183 & Tanzania & $\#$ & N/A & $\mathrm{A}^{*}$ & N/A & NM & Morogoro & Kasanga et al., 2012 \\
\hline 184 & Tanzania & \# & N/A & $A^{*}$ & $\mathrm{~N} / \mathrm{A}$ & NM & Pwani & Kasanga et al., 2012 \\
\hline
\end{tabular}

Note. NM, Not mentioned; * samples analysed using Ag-ELISA; N/A, Non applicable; \# Year range 2003 and 2010.

\section{Results}

\subsection{Temporal Serotype Distribution}

Five FMDV serotypes; O, A, SAT 1, SAT 2 and SAT 3, were detected in Uganda, whereas four serotype O, A, SAT 1 and SAT 2, were detected in Tanzania between 2003 and 2015 (Balinda et al., 2010a; Balinda et al., 2010b; 
Kasanga et al., 2012; Kasambula et al., 2012; Sallu et al., 2014; Namatovu et al., 2015; Kasanga et al., 2015; Dhikusooka et al., 2015; Dhikusooka et al., 2016).

\subsubsection{Serotype O}

Between 2003 and 2015, serotype $\mathrm{O}$ was reported in 10 out of the 13 years in both Uganda and Tanzania. In 2005, 2006, 2008 and 2009, 2010 in Uganda, all the outbreaks were associated with only serotype O. From 2003 to 2015, alongside one other serotypes, serotype $O$ was cited again in all the outbreaks that occurred in the country (Figure 1 and Table 1), making it the most predominant serotype in Uganda between 2003 and 2015 . The situation was closely similar in Tanzania, where serotype O outbreaks were reported annually except for 2013, 2014 and 2015. It was also observed that in Tanzania, serotype O occurred alongside at least one other serotype (SAT 1 and SAT 2) (Figure 2). This review suggests that serotype $\mathrm{O}$ was accountable for most of the outbreaks in Uganda stretching from south western Uganda to Kaabong in far eastern part of Uganda (Figures 1 and 3). While, in Tanzania, the distribution of serotype $\mathrm{O}$ was evenly spread across the country and occurred in the regions of Mwanza, Kagera, Mara, Shinyanga, Tabora, Arusha, Tanga, Da es Salaam, Mtwara, Lindi, Ruvuma, Iringa, Morogoro, Pwani, Mbeya, Njombe and Rukwa. The review revealed that the serotype O viruses that were published between 2003 and 2015 from both countries, all belonged to topotype EA-2.

\subsubsection{Serotype A}

The observations from this review indicated that serotype A was detected in Uganda in 2013 and 2014, while in Tanzania it was detected in 2008, 2009 and 2012. Additionally, the findings from this review reveal that serotype A was detected only in the western and central parts of Uganda in the districts of Kirihura and Wakiso. There were no outbreaks of serotype A detected in the eastern and northern parts of Uganda. However, in Tanzania, although the serotype occurred a few times, it was distributed throughout the regions of the country in the regions of Kagera, Mara, Arusha, Kigoma, Tabora, Rukwa, Njombe, Iringa, Dodoma, Morogoro, Pwani, Mtwara and Dar es Salaam (Figure 3). We additionally observed that the Ugandan and Tanzanian FMD serotype A all belonged to genotype 1 (G-1) within the AFRICA topotype, to which the vaccine strain A/K5/1980 that was being used belonged.

\subsubsection{Serotype SAT 1}

The SAT 1 viruses in Uganda was detected in 2011 and 2015 in cattle from Kasese and Kirihura, districts respectively. The serotype was also detected in buffalo in 2007 from Lake Mburo National Park in Mbarara district. From these findings, the review shows that in Uganda, the SAT 1 viruses were found only in the south western region of the country and there was no detection of SAT 1 viruses in the central, northern and eastern regions. In Tanzania, SAT 1 viruses were detected every year from 2003 up to 2014. The SAT 1 viruses were distributed only along the eastern part of Tanzania and were found in the regions of Mara, Manyara, Morogoro and Dar es Salaam. We further observed in this review that all the Tanzanian SAT 1s belonged to the topotypeI also called North-Western Zimbabwe (NWZ) whereas the SAT 1s of Uganda belonged to topotype IV also known as East Africa-1.

\subsubsection{Serotype SAT 2}

Serotype SAT 2 was detected in samples from Ugandan samples collected in 2004, 2013 and 2014 from the districts of Kiboga in the central and Isingiro and Kirihura in the western region of the country. No SAT 2 viruses were detected in the eastern and northern regions of Uganda. In Tanzania, SAT 2 was confirmed in all the nine years from 2003 to 2012. The review highlighted that the distribution of SAT 2 in Tanzania was approximately correlated to the distribution of wildlife reservoirs (Figure 3). More than half of the regions that harboured a national park or game reserve had recorded an outbreak by SAT 2. Detected SAT 2 viruses were from the regions of Kagera, Mara, Arusha, Tabora, Rukwa, Mbeya, Singida, Manyara, Kilimanjaro, Morogoro, Pwani, Kigoma, Iringa, and Njombe. It was further observed that the SAT 2 FMDV from Uganda and Tanzania belonged to different topotypes, I and IV respectively.

\subsubsection{Serotype SAT 3}

Between 2003 and 2015, this serotype was never detected in Tanzania but was isolated in Uganda in 2013 from Kasese district near the Queen Elizabeth National Park, 16 years after it was last isolated from an African Buffalo. 


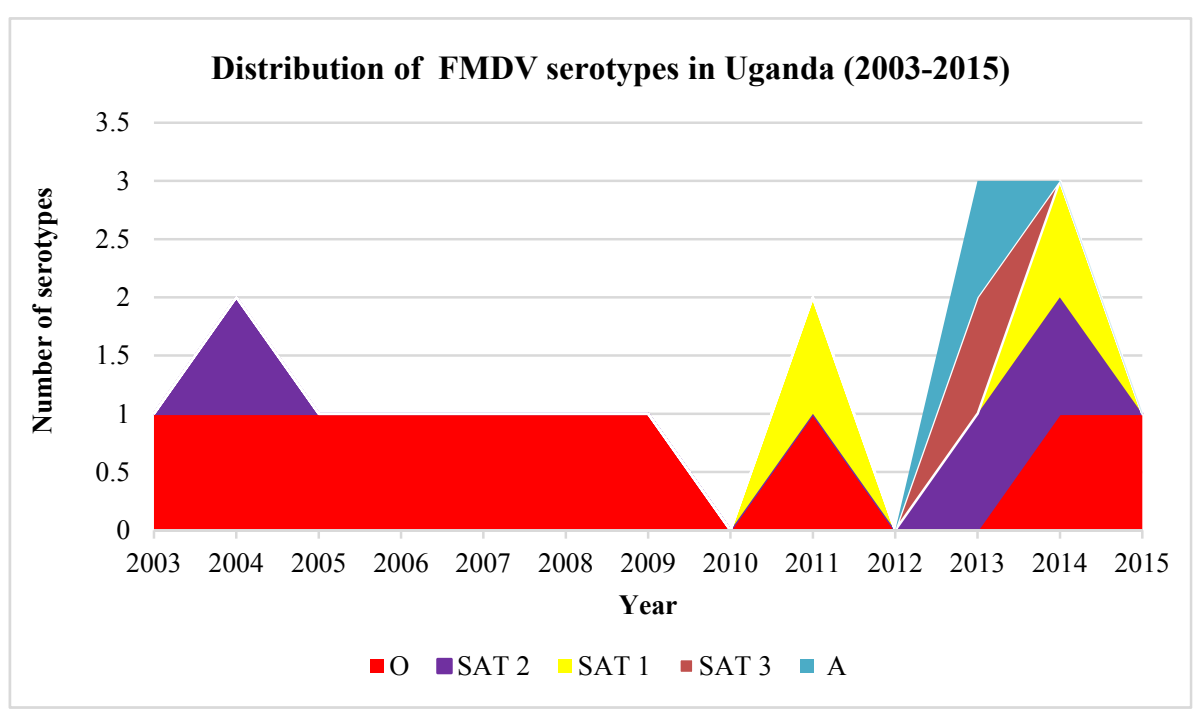

Figure 1. Distribution of FMDV serotypes in Uganda

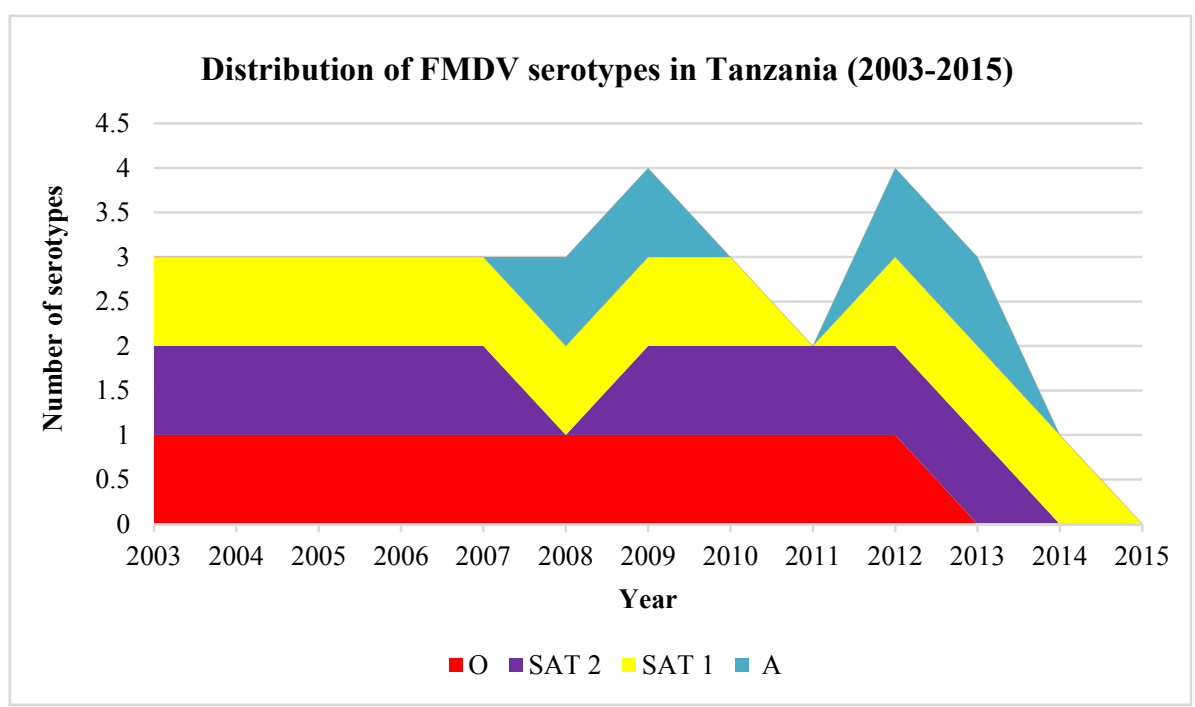

Figure 2. Distribution of FMDV serotypes in Tanzania

\subsection{Spatial Distribution}

From this review, it was observed that between 2003 and 2015, the western and central regions of Uganda registered more outbreaks than the northern and eastern regions. The serotype diversity was highest in western Uganda with five serotypes (O, A, SAT 1, SAT 2 and SAT 3) documented. The central region followed with three serotypes namely, O, A, and SAT 2 and, the eastern region and northern regions had only serotype $\mathrm{O}$ in circulation. The outbreaks in the northern region were observed in the districts around the Lake Kyoga basin. The west Nile region had no outbreaks recorded for the entire study period (Figure 3). 


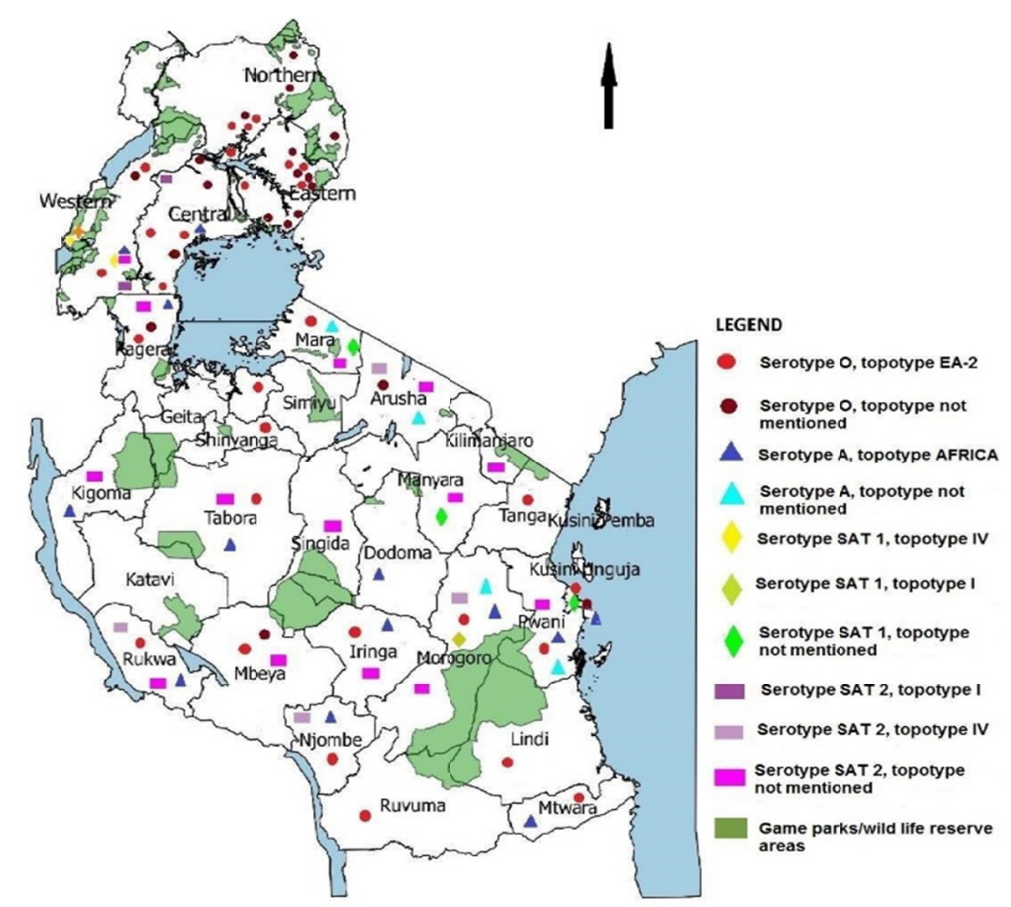

Figure 3. Spatial distribution of FMDV serotypes in Uganda and Tanzania (2003-2015)

In Tanzania, between 2003 and 2015, there was a wide distribution of multiple serotypes (Figure 3). Regions such as Kagera, Pwani, Morogoro, Arusha and Iringa registered more outbreaks than other regions during the eleven years. The highest serotype diversity was realised in Morogoro, Mara and Pwani which had all the four serotypes, (O, A, SAT 1 and SAT 2). Rukwa, Iringa, Arusha, Dar es Salaam, Njombe and Tabora regions followed with three serotypes each (Figure 3). Other areas like Geita and Katavi did not have any FMD outbreaks reported according to the reviewed publications.

\section{Discussion}

Inadequate documentation and characterisation of the occurrence of FMD outbreaks in Uganda and Tanzania creates deficiency of information for effective disease control within the East African region (Namatovu et al., 2013; Dhikusooka et al., 2015). In this review, using the available literature, occurrence of the different serotypes in Uganda and Tanzania was determined. From published literature, differences in the topotypes of circulating serotypes in each country was demonstrated.

\subsection{Serotype $O$}

Previous studies have shown serotype $\mathrm{O}$ to be the most prevalent serotype circulating both in Uganda and Tanzania (Balinda et al., 2010a; Kasambula et al., 2012; Kasanga et al., 2015; Sallu et al., 2014) which is in agreement with the observations from this review. In Uganda, serotype $\mathrm{O}$ was detected from the south western part of Uganda to the far-east in Kaabong district, illustrating its wide distribution across the country. Other studies in Uganda have demonstrated the dominance of this serotype (Balinda et al., 2010a; Kasambula et al., 2012; Kerfua, Isubikalu, Ademun-Okurut, Muwanika, \& Masembe, 2013; Namatovu et al., 2015) in the different regions. In Tanzania, the distribution of serotype $\mathrm{O}$ was fairly even across the country, as supported by previous studies. Sallu et al. (2014) observed that serotype $\mathrm{O}$ was wide spread in Tanzania and was responsible for most of the outbreaks between 2003 and 2008. Kasanga et al. (2012) similarly reported that serotype O was widespread throughout Tanzania except for some of the central parts of the country.

Although topotype EA-2 was found dominant in Uganda and Tanzania, this is contrary to what has been observed previously in the 1980s and 90s when topotype EA-1 was circulating in both countries (Vosloo, 2002; Balinda et al., 2010a). The disappearance of topotype EA-1 remains mysterious but probably shows that the topotype may be approaching extinction in the two countries or its detection overlooked. However, the predominant circulation of the topotype EA-2 in Uganda and Tanzania has implications for FMD control because the current vaccine strain (O/KEN/77/78) in use belongs to EA-1. Earlier studies by Kitching et al. (2007) have 
argued that wide antigenic differences between the vaccines and circulating strains may result in a failure of the vaccine to generate protective antibodies. Thus the need for constant vaccine evaluation studies.

\subsection{Serotype A}

This paper demonstrated the rare detection of serotype $\mathrm{A}$ in both countries for the period covered by the review. Namatovu et al. (2015) highlighted the infrequent detection of this serotype in Uganda. Before its detection in 2013, the serotype has been last documented in 2002 (Namatovu et al., 2015). While in Tanzania, serotype A was regularly detected in the northern parts of Tanzania from 1954 up to 1971, but disappeared for over 30 years until its detection in 2008 in Iringa (Kasanga et al., 2012). The reason for the rare appearance of serotype A is still not well understood but its appearance and disappearance has been highlighted in the Asian continent as well (Kitching, 2005). Casey-Bryars et al. (2018) observed that serotypes were spreading over landscapes by waves and the distribution patterns were not random. The authors argued that certain serotypes were dominant during outbreaks for a specific time, after which they did not appear immediately (Casey-Bryars et al., 2018). This could be the same case for serotype A virus outbreak patterns in both Uganda and Tanzania. Never the less, more research is required in understanding the serotype A patterns in both countries in order to inform better control strategies. The spatial distribution of serotype A in Uganda demonstrates its confinement to the western and central parts of Uganda, suggesting certain epidemiological factors may be playing a role. The similarity in serotype A, lineages between the two countries may be indicative of FMDV serotype A spread between the two countries given the proximity between the south western region of Uganda and the northern regions of Tanzania. Despite the few years in which serotype A was detected in Tanzania, its distribution in the country was roughly even throughout. This could mean that the serotype easily spread across the different regions in the country probably due to factors such as uncontrolled animal movement, poor uptake of vaccination programs, and lack of biosecurity measures in place among others

Previous studies have shown that older Ugandan serotype A viruses isolated in the 60s belonged to genotype G-VII of AFRICA topotype to which the vaccine strain K35/1980 belongs (Namatovu et al., 2015). Although, the recent viruses belong to same topotype as the vaccine strain, Namatovu et al. (2015) highlighted antigenic differences between them that could affect the efficacy of the vaccine. Additionally, previous research has also shown that serotype A is a highly diverse serotype that is constantly evolving. This constant evolution is able give rise to new variants that may not be so closely antigenically related (Kitching, 2005; Kasanga et al., 2015; Sallu et al., 2014). Consequently these variants may complicate the control of FMD by vaccination.

\subsection{Serotype SAT 1}

The observed trend of the high detection of SAT 1 in Tanzania compared to that in Uganda, may highlight differences in surveillance and reporting systems, SAT 1 incursions or/and submission of samples for diagnosis. One of the challenges in comprehending the epidemiology and risk of FMD in endemic settings, is the low submission of samples both to the regional and world reference laboratories (Namatovu et al., 2013). Other reasons for the observed disparity in the frequency of reported SAT 1 occurrence in Uganda and Tanzania may be due to underreporting or limited appropriate diagnostic tools in Uganda (Dhikusooka et al., 2016; Kerfua et al., 2018).

Previously conducted studies have also shown that SAT 1 Ugandan isolates were significantly different from other SAT 1s from Tanzania and Kenya (Dhikusooka et al., 2016), suggesting that the SAT 1 virus strains from Uganda strains are most likely geographically confined to Uganda (Sangula et al., 2010). This could explain the observed differences in topotypes presented. All the Tanzanian SAT 1s belonged to the toptype I (NWZ) (Kasanga et al., 2015), whereas those of Uganda belonged to topotype IV (East Africa-1). According to Sangula et al. (2010), SAT 1 viruses in East Africa have evolved from two independent lineages from South Africa. Ugandan viruses were found to be related to West African and Sudanese viruses while Tanzanian and Kenyan SAT 1 viruses were found to be closer to each other (Kasanga et al., 2015). These observations could aid in explaining the spatial distribution of Tanzanian SAT 1s which were found in the eastern part of the country that is close to Kenya (Figure 3).

The TAN/155/71 vaccine strain that belongs to the toptype I (NWZ) has been consistently used in controlling FMD spread in the region. Since the vaccine strain belongs to a different topotype from the Ugandan strain, there may be no cross protection between these strains thus a lot of implications for FMD control. This indicates the need for more studies in Uganda on vaccine evaluation in order to ascertain the protection elicited by the TAN/155/71 vaccine strain.

Dhikusooka et al. (2016) reported silent manifestation of clinical signs in the cattle from which the SAT 1 viruses were isolated. This kind of scenario presents challenges to the clinical based reporting systems (Namatovu et al., 
2013), where most reported FMD cases in Uganda and Tanzania were shown to rely on clinical manifestations of the disease instead of laboratory diagnosis (Muleme et al., 2012; Kerfua et al., 2018).

\subsection{Serotype SAT 2}

The occasional detection of SAT 2 in Uganda may be attributed to limited outbreak surveillance and may not reflect the true representation of the situation in the country. It may also be that SAT 2 viruses were rare in Uganda during the reviewed period. However, in Tanzania, the observed widespread and frequent occurrence of SAT 2 could be attributed to regular sample diagnosis or/and the presence of high numbers of wildlife reservoirs. In this review, it was observed that the distribution of SAT 2 in Tanzania was roughly correlated to the distribution of wild life reservoirs (Figure 2). In Uganda, though, the distribution of SAT 2 viruses was mostly confined to western region of Uganda. However, the eastern part of the country that has three major national parks harbouring buffalos (Mt Elgon, Kidepo and Pian Upe), did not have SAT 2s detected. The association of SAT 2 outbreaks with wildlife-livestock interphases were observed in studies carried out in the southern and eastern Africa, and the African Buffalo has been connected with the SAT carrier status, and implicated in virus exchange with cattle (Woodsbury, 1995; Vosloo et al., 2005; Kasanga et al., 2012; Ayebazibwe et al., 2010a; Jori et al., 2016). However, other studies have shown that it's not always the case that buffalos are responsible for SAT 2 incursions (Brito et al., 2016). Nevertheless, reasons for this distribution pattern of SAT 2 in Tanzania and Uganda still remain poorly understood (Kasanga et al., 2012; Sallu et al., 2014).

According to Balinda et al. (2010b), the 2010 SAT 2 Ugandan viruses were different from viruses that were isolated in years of 1975, 1995, 1998 and 2002. We suggest that the viruses could have evolved along different lines and raises questions on whether they could have emerged from different buffalo populations. However, the limited number of SAT 2 FMD virus sequences from Uganda limits the comprehensive phylogenetic analysis of FMDVs SAT 2 from both cattle and buffalo. Furthermore, disparity in topotypes of the SAT 2 FMDV from Uganda and Tanzania either implies that SAT 2 viruses are not spreading between country or that there may be under reporting of SAT 2 incursions in Uganda. According to Balinda et al. (2010b) and Namatovu et al. (2013), Uganda has reported two virus lineages of SAT 2 FMDVs. However, the most recent isolates have been observed to belong to a similar lineage as the vaccine strain (K52/84). Namatovu et al. (2015) further demonstrated that vaccine strain had significant genetic diversity with the most recent circulating SAT 2 virus, underscoring a need for vaccine matching studies to be carried out in both Uganda and Tanzania.

\subsection{Serotype SAT 3}

Previous SAT 3 isolates from Uganda were only from buffalo (Kalema-Zikusoka et al., 2005; Ayebazibwe et al., 2010a). The SAT 3 isolate was retrieved from a calf that had been grazing in the Queen Elizabeth National Park (QENP). The findings from the study by Dhikusooka et al. (2015), presents a new challenge for continued surveillance in the livestock wildlife interphase areas both in Uganda and Tanzania.

The SAT 3 isolate had a 19\% a nucleotide difference with buffalo FMD virus UGA/2/97 that was grouped in topotype IV. However, Dhikusooka et al. (2015), recommended that the isolated SAT 3 virus from Uganda should be categorised within a single topotype V. Currently, there is no vaccine strain that incorporates the SAT 3 serotype. This calls for further vaccine development and other strategic controls.

\section{Conclusion}

This review presents similarities and differences in the distribution of FMD serotypes in Uganda and Tanzania. It also highlights regions with the highest serotype diversity and those with the least diversity. In addition, this review has shown that there are differences between the circulating virus serotypes/topotypes and the vaccine strains currently in use in both countries. Although, underreporting of outbreaks and limited resources for diagnosis hinder the true picture of the serotype distribution in endemic countries, these findings still provide information important for strategizing control of FMD. For example, efforts for control can be specifically targeted to an area based on the prevalent serotypes and the serotype diversity.

This review recommends routine characterisation of circulating viruses, further research on FMD risk factors for certain serotypes, vaccine evaluation studies and implementation of harmonised regional FMD control programmes.

\section{Acknowledgements}

We acknowledge the Bill \& Melinda Gates Foundation for funding this study through the Program for Enhancing Health and Productivity in Livestock (PEHPL). 


\section{References}

Ahmed, H. A., Salem, S. A., Habashi, A. R., Arafa, A. A., Ag-gour, M. G., Salem, G. H., ... King, D. P. (2012). Emergence of foot-and-mouth disease virus SAT 2 in Egypt during 2012. Transboundary and Emerging Diseases, 59, 476-481. https:// doi.org/10.1111/tbed.12015

Ayebazibwe, C., Mwiine, F. N., Tjørnehøj, K, Balinda, S. N., Muwanika, V. B., Ademun Okurut, A. R., ... Alexandersen, S. (2010a). The role of African buffalos (Syncerus caffer) in the maintenance of foot-and-mouth disease in Uganda. BMC Veterinary Research, 6, 54. https://doi.org/10.1186/17466148-6-54

Ayebazibwe, C., Tjørnehøj, K., Mwiine, F. N., Muwanika, V. B., Ademun Okurut, A. R., Siegismund, H. R., \& Alexandersen, S. (2010b). Patterns, risk factors and characteristics of reported and perceived foot-and-mouth disease (FMD) in Uganda. Tropical Animal Health and Production, 42(7), 1547-1559. https://doi.org/10.1007/s11250-010-9605-3

Balinda, S. N., Sangula, A. K., Heller, R., Muwanika, V. B., Belsham, G. J., Masembe, C., \& Siegismund, H. R. (2010a). Diversity and transboundary mobility of serotype $\mathrm{O}$ foot-and-mouth disease virus in East Africa: Implications for vaccination policies. Infection, Genetics and Evolution, 10(7), 1058-1065. https://doi.org/ 10.1016/j.meegid.2010.06.017

Balinda, S. N., Belsham, G. J., Masembe, C., Sangula, A. K., Siegismund, H. R., \& Muwanika, V. B. (2010b). Molecular characterization of SAT 2 foot-and-mouth disease virus from post-outbreak slaughtered animals: implications for disease control in Uganda. Epidemiology and Infection, 138, 1204-1210. https://doi.org/ $10.1017 / \mathrm{S} 0950268809991427$

Baluka, S. A. (2016) Economic effects of food and mouth disease outbreaks along the cattle marketing chain in Uganda. Veterinary World, 9, 544-553. https:// doi.org/10.14202/vetworld.2016.544-553

Bari, F. D., Parida, S., Tekleghiorghis, T., Dekker, A., Sangula, A., Reeve, R., ... Mahapatra, M. (2014) Genetic and antigenic characterisation of serotype A FMD viruses from East Africa to select new vaccine strains. Vaccine, 32(44), 5794-5800. https://doi.org/10.1016/j.vaccine.2014.08.033

Bengis, R., Thomson, G., Hedger, R., De Vos, V., \& Pini, A. (1986). Foot-and-mouth disease and the African buffalo (Syncerus caffer). L. Carriers as a source of infection for cattle. Onderstepoort Journal of Veterinary Research, 53, 69-73.

Bengis, R. (2005). Transfrontier conservation area initiatives in Sub-Saharan Africa: Some animal health challenges. In S. A. Osofsky, S. Cleaveland, W. Karesh, M. Kock, P. Nyhus, \& A. Yang (Eds.), Conservation and Development Interventions at the Wildlife/Livestock Interface: Implications for Wildlife, Livestock and Human Health (pp. 15-19). Occasional paper IUCN SSC, Gland, Switzerland and Cambridge, UK.

Brito, B. P., Jori, F., Dwarka, R., Maree, F. F., Heath, L., \& Perez, A. M. (2016). Transmission of Foot-and-Mouth Disease SAT2 Viruses at the Wildlife-Livestock Interface of Two Major Transfrontier Conservation Areas in Southern Africa. Frontiers in Microbiology, 7, 528. https://doi.org/10.3389/fmicb. 2016.00528

Bruckner, G. K., Vosloo, W., Du Plessis, B. J. A., Kloeck, P. E. G., Cannoway, L., Ekron, M. D., ... Mogajane, M. E. (2002). Foot-and-mouth Disease: The experience of South Africa. Scientific and Technical Reviews of office International des Epizooties, 21(3), 751-754. https://doi.org/ 10.20506/rst.21.3.1368

Casey-Bryars, M., Reeve, R., Bastola, U., Knowles, N. J., Auty, H., Bachanek-Bankowska, K., ... Lembo, T. (2018). Waves of endemic foot-and-mouth disease in eastern Africa suggest feasibility of proactive vaccination approaches. Nature Ecology and Evolution, 9, 1449-1457. https://doi.org/10.1038/s41559-0180636-x

Chitray, M., de Beer, T. A., Vosloo, W., \& Maree, F. F. (2014). Genetic heterogeneity in the leader and $\mathrm{P} 1$-coding regions of foot-and-mouth disease virus serotypes $\mathrm{A}$ and $\mathrm{O}$ in Africa. Archives in Virology, 159(5), 947-61. https://doi.org/ 10.1007/s00705-013-1838-9

Christensen, L. S., Okorut, R., Tjørnehøj, K., Normann, P., Sørensen, K. J., \& Esau, M. (2004). Characterisation of a new type O lineage of FMDV from Uganda with atypical clinical manifestations in domestic cattle. Report of the Open Session of the Research Group of the Standing Technical Committee of the European Commission for the control of Foot-and-Mouth disease (pp. 159-162). Crete, Greece. 
Dhikusooka, M. T., Ayebazibwe, C., Namatovu, A., Belsham, G. J., Siegismund, H. R., Wekesa, S. N., \& Tjørnehøj, K. (2016). Unrecognized circulation of SAT 1 foot-and-mouth disease virus in cattle herds around Queen Elizabeth National Park in Uganda. BMC Veterinary Research, 12, 5. https://doi.org/ 10.1186/s12917-015-0616-1

Dhikusooka, M. T., Tjørnehøj, K., Ayebazibwe, C., Namatovu, A., Ruhweza, S., Siegismund, H. R., \& Belsham, G. J. (2015). Foot-and-mouth disease virus serotype SAT 3 in long-horned Ankole calf, Uganda. Emerging Infectious Diseases, 21(1), 111-114. https://doi.org/10.3201/eid2101.140995

Domingo, E., Mateu, M. G., Martinez, M. A., Dopazo, J., Moya, A., \& Sobrino, F. (1990). Genetic variability and antigenic diversity of foot-and-mouth disease virus. In E. Kurstak, R. G. Marusyk, S. A. Murphy, \& M. H. V. Van Regenmortel (Eds.), Applied Virology Research, 2, Virus Variation and Epidemiology (pp. 233-266). New York: Plenum. https://doi.org/10.1007/978-1-4757-9271-3_15

Domingo, E., Baranowski, E., \& Escarmis, S. (2002). Foot-and-mouth disease. Journal of Comparative Immunology, Microbiology and Infectious Disease, 25(5), 297-308. https://doi.org/10.1016/s0147-9571 (02)00027-9

Donaldson, A. I., Gloster, J., Harvey, L. D. J., \& Deans, D. H. (1982). Use of prediction models to forecast and analyse airborne spread during the foot-and-mouth disease outbreaks in Brittany, Jersey and the Isle of Wight in 1981. Veterinary Record, 110, 53-57. https://doi.org/10.1136/vr.110.3.53

Donaldson, A. I., \& Alexandersen, S. (2002). Predicting the spread of foot-and-mouth disease by airborne virus. Revue scientifique et technique (International Office of Epizootics), 21, 569-575. https://doi.org/10.20506/ rst.21.3.1362

FAO. (2011). The Progressive Control Pathway for FMD control (PCP-FMDFMD): Principles, Stage Descriptions and Standards. Retrieved from http://www.fao.org/ag/againfo/commissions/docs/PCP-FMD/ PCP-FMD-26012011.pdf

FAO. (2018). The global foot-and-mouth disease control strategy. Retrieved from https:/www.fao.org/3/ I9857EN/i9857en.PDF

Fèvre, E. M., Bronsvoort, B. M., Hamilton, K. A., \& Cleaveland, S. (2006). Animal movements and the spread of infectious diseases. Trends in Microbiology, 14(3), 125. https://doi.org/10.1016/j.tim.2006.01.004

Fry, E. E., Newman, J. W. I., Curry, S., Najjam, S., Jackson, T., Blakemore, W., ... Stuart, D. I. (2005). Structure of foot-and-mouth disease virus serotype $\mathrm{A}_{10} 0_{61}$ alone and complexed with oligosaccharide receptor: receptor conservation in the face of antigenic variation. Journal of General Virology, 86, 1909-1920. https://doi.org/10.1099/vir.0.80730-0

Jamal, S. M., \& Belsham, G. J. (2013). Foot-and-mouth disease: past, present and future. Veterinary Research, 44, 116. https://doi.org/10.1186/1297-9716-44-116

James A.D., \& Rushton, J. (2002). The economics of foot-and-mouth disease. Revue Scientifique et Technique (International Office of Epizootics), 21, 637-644. https://doi.org/10.20506/rst.21.3.1356

Jori, F., Vosloo, W., Du Plessis, B., Bengis, R., Brahmbhatt, D., Gummow, B., \& Thompson, G. R. (2009). A qualitative risk assessment of factors contributing to foot and mouth disease outbreaks in cattle along the western boundary of the Kruger National Park. Revue Scientifique et Technique (International Office of Epizootics, 28, 917-931. https://doi.org/10.20506/rst.28.3.1932

Kalema-Zikusoka, G., Bengis, R. G., Michel, A. L., \& Woodford, M. H. (2005). A preliminary investigation of tuberculosis and other diseases in African buffalo (Syncerus caffer) in Queen Elizabeth National Park, Uganda. Onderstepoort Journal of Veterinary Research, 72, 145-51. https://doi.org/10.4102/ojvr.v72i2.210

Kasambula, L., Belsham, G. J., Siegismund, H. R., Muwanika, V. B., Ademun-Okurut, A. R., \& Masembe, C. (2012). Serotype identification and VP1 coding sequence analysis of foot-and-mouth disease viruses from outbreaks in eastern and northern Uganda in 2008/9. Transboundary and Emerging Diseases, 59, 323-330. https://doi.org/10.1111/j.1865-1682.2011.01276.x

Kasanga, C. J., Sallu, R., Kivaria, F., Mkama, M., Masambu, J., Yongolo, M., .. Rweyemamu, M. M. (2012). Foot-and-mouth disease virus serotypes detected in Tanzania from 2003 to 2010: Conjectured status and future prospects. Onderstepoort Journal of Veterinary Research, 79(2), 462. https://.doi.org/10.4102/ ojvr.v79i2.462 
Kasanga, C. J., Wadsworth, J., Mpelumbe-Ngeleja, C. A. R., Sallu, R., Kivaria, F., Wambura, P. N., ... King, D. P. (2015). Molecular Characterization of Foot-and-Mouth Disease Viruses Collected in Tanzania Between 1967 and 2009. Transboundary and Emerging Diseases, 16,19-29. https://doi.org/ 10.1111/tbed.12200

Kerfua, S. D., Isubikalu, P., Ademun-Okurut, A. R., Muwanika, V. B., \& Masembe, C. (2013). Molecular characterization of serotype $\mathrm{O}$ foot-and-mouth disease virus from pigs: Implications for multispecies approach to disease control in Uganda. African Journal of Biotechnology, 12, 19.

Khan, S. K., Regina Kunz, R., Kleijnen, J., \& Antes, G. (2003). Five steps to conducting a systematic review. Journal of the Royal Society of Medicine, 96, 118-121. https://doi.org/10.1258/jrsm.96.3.118

Kitching, R. P. (2002). Clinical variation in foot-and-mouth disease: cattle. Revue Scientifique et Technique de l'Office International des Epizooties, 21(3), 499-504. https://doi.org/10.20506/rst.21.3.1343

Kitching, R. P. (2005). Global Epidemiology and Prospects for Control of Foot-and-Mouth Disease. In B. W. Mahy (Ed.), Foot-and-mouth disease virus (pp. 133-148). Manitoba: Springer. https://doi.org/10.1007/ 3-540-27109-0_6

Kitching, P., Hammond, J., Jeggo, M., Charleston, B., Paton, D., Rodriguez, L., \& Heckert, R. (2007). Global FMD control-Is it an option? Vaccine, 25(30), 5660-5664. https://doi.org/10.1016/j.vaccine.2006.10.052

Kivaria, F. M. (2003). Foot-and-mouth disease in Tanzania: An overview of its national status. The Veterinary Quaterly, 25(2), 72-78. https://doi.org/10.1080/01652176.2003.9695147

Knowles, N. J., \& Samuel, A. R. (2003). Molecular epidemiology of foot-and-mouth disease virus. Virus Research, 91, 65-80. https://doi.org /10.1016/s0168-1702(02)00260-5

Knowles, N. J., Samuel, A. R., Davies, P. R., Midgley, R. J., \& Valarcher, J. F. (2005). Pandemic strains of foot-and-mouth disease virus serotype O. Emerging Infectious Diseases, 11, 1887-1893. https://doi.org/ 10.3201/eid1112.050908

Logan, G., Freimanis, G. L., King, D. J., Valdazo-González, B., Bachanek-Bankowska, K., Sanderson, N. D., ... Cottam, E. M. (2014). A universal protocol to generate consensus level genome sequences for foot-and-mouth disease virus and other positive-sense polyadenylated RNA viruses using the Illumina MiSeq. BMC Genomics, 15, 828. https://doi.org/10.1186/1471-2164-15-828.

Maree, F. F., Kasanga, C. J., Scott, K. A., Opperman, P. A., Chitray, M., Sangula, A. K., ... Rweyemamu, M. M. (2014) Challenges and prospects for the control of foot-and-mouth disease: An African perspective. Veterinary Medicine: Research and Reports, 5, 119-138. https://doi.org/10.2147/VMRR.S62607

Maziku, M., Mruttu, H., \& Gebru, G. (2016). Animal health strategy and vision for Tanzania. Nairobi, Kenya: Tanzania Ministry of Agriculture, Livestock and Fisheries and International Livestock Research Institute. Retrieved from http://www.agriknowledge.org/file_downloads/41687h474

Miguel, E., Grosbois, V., Caron, A., Boulinier, T., Fritz, H., Cornelis, D., ... de Garine-Wichatitsky, M. (2013): Contacts and foot and mouth disease transmission from wild to domestic bovines in Africa. Ecosphere, 4, art51. https://doi.org/10.1890/ES12-00239.1

Muleme, M., Barigye, R., Margaret, L., Khaitsa, M. L., Berry, E., Wamono, A. W., \& Ayebazibwe, A. (2012). Effectiveness of vaccines and vaccination programs for the control of foot and mouth disease in Uganda, 2001-2010. Tropical Animal Health and Production, 45(1), 35-43. https://doi.org/10.1007/s11250-0120254-6

Mwanandota, J. J., Kasanga, C. J., Yongolo, M., Sallu, R., \& Mkama, M. (2013). Spatial and temporal distribution of foot-and-mouth disease virus in the eastern zone of Tanzania. Tanzania Veterinary Journal, $28(2), 39-46$.

Mwiine, F. N., Ayebazibwe, C., Olaho-Mukani, W., Alexandersen, S., Balinda, S.N., Masembe, C., ... Tjørnehøj, K. (2010). Serotype-specificity of antibodies against foot and mouth disease virus in cattle in selected districts in Uganda. Transboundary and Emerging Diseases, 57(5), 365-74. https://doi.org/10.1111/ j.1865-1682.2010.01157.x

Namatovu, A., Wekesa, S. N., Tjørnehøj, K., Dhikusooka, M. T., Muwanika, V. B., Siegsmund, H. R., \& Ayebazibwe, C. (2013). Laboratory capacity for diagnosis of foot-and-mouth disease in Eastern Africa: implications for the progressive control pathway. BioMedCentre, Veterinary Research, 9, 13. https://doi.org/ $10.1186 / 1746-6148-9-19$ 
Namatovu, A., Tjørnehøj, K., Belsham, G. J., Dhikusooka., M. T., Wekesa, S. N., Muwanika, V. B., ... Ayebazibwe, C. (2015). Characterization of foot-and-mouth disease viruses (FMDVs) from Ugandan cattle outbreaks during 2012-2013: Evidence for circulation of multiple serotypes. Plos One, 10, e0114811. https://doi.org/10.1371/journal.pone.0114811

OIE. (2012). Foot and mouth disease, In OIE Manual of diagnostic tests and vaccines for terrestrial animals (pp. 190-216). Office International des Epizooties, Paris, France. Retrieved from https://www.oie.int/doc/ged/ D7710.PDF

OIE. (2017). Foot-and-mouth disease. Retrieved from http://www.oie.int/animal-health-in-the-world/officialdisease-status/fmd

Railey, A. F., Lembo, T., Palmer, G. H., Shirima, G. M., \& Marsh, T. L. (2018). Spatial and temporal risk as drivers for adoption of foot-and-mouth disease vaccination. Vaccine, 36(33), 5077-5083. https://doi.org/ 10.1016/j.vaccine.2018.06.069

Rweyemamu, M., Roeder, P., Mackay, D., Sumpton, K., Brownlie, J., Leforban, Y., ... Saraiva, V. (2008). Epidemiological patterns of foot-and-mouth disease worldwide. Transboundary and Emerging Diseases, 55, 57-72. https://doi.org/10.1111/j.1865-1682.2007.01013.x

Sahle, M. (2004). An epidemiological study on the genetic relationships of foot and mouth disease viruses in East Africa ( $\mathrm{PhD}$ thesis, University of Pretoria, Pretoria, South Africa). Retrieved from https://repository.up.ac.za/bitstream/handle/2263/27222/Complete.pdf?sequence=5

Sahle, M., Dwarka, R. M., Venter, E. H., \& Vosloo, W. (2007). Comparison of SAT-1 foot-and-mouth disease virus isolates obtained from East Africa between 1971 and 2000 with viruses from the rest of sub-Saharan Africa. Archives of Virology, 152, 797-804. https://doi.org/10.1007/s00705-006-0893-x

Sangula, A. K., Belsham, G. J., Muwanika, V. B., Heller, R., Balinda, S. N., \& Siegismund, H. R. (2010). Co-circulation of two extremely divergent serotype SAT 2 lineages in Kenya highlights challenges to foot-and-mouth disease control. Archives of Virology, 155, 1625-1630. https://doi.org/10.1007/s00705010-0742-9

Sangula, A. K., Siegismund, H. R., Belsham, G. J., Balinda, S. N., Masembe, C., \& Muwanika, V. B. (2011). Low diversity of foot-and-mouth disease serotype $\mathrm{C}$ virus in Kenya: Evidence for probable vaccine strain re-introductions in the field. Epidemiology and Infection, 139(2), 189-196. https://doi.org/10.1017/ S0950268810000580

Sallu, R. S., Kasanga, C. J., Mathias, M., Yongolo, M., Mpelumbe-Ngeleja, C., Mulumba, M., ... King, D. (2014). Molecular survey for foot-and-mouth disease virus in livestock in Tanzania, 2008-2013, Onderstepoort Journal of Veterinary Research, 81(2), a736. https:// doi.org/10.4102/ojvr.v81i2.736.

Stanway, G., Brown, F., Christian, P., Hovi, T., Hypia, T., King, A. M. Q., ... Skern, T. (2005). Family Picornaviridae. In C. M. Fauquet, M. A. Mayo, J. Maniloff, U. Desselberger, \& L. A., Ball (Eds.), Virus Taxonomy. Eighth Report of the International committee on Taxonomy of viruses (pp. 757-778). London: Elsevier/Academic Press.

Stenfeldt, C., Diaz-San Segundo, F., de Los Santos, T., Rodriguez, L. L., \& Arzt, J. (2016). The Pathogenesis of Foot-and-Mouth Disease in Pigs. Frontiers in Veterinary Science, 3, 41. https://doi.org/10.3389/fvets. 2016.00041

Sutmoller, P., \& Casas, O. R. (2002). Unapparent foot and mouth disease infection (sub-clinical infections and carriers): Implications for control. Revue Scientifique et Technique de l'Office International des Epizooties, 21(3), 519-529. https://doi.org/10.20506/rst.21.3.1366

Thompson, G. R. (1994). Foot-and-mouth disease. In J. A. W. Coetzer, G. R. Thompson, \& R. C. Tustin (Eds.), Infectious diseases of livestock with special reference to southern Africa (pp. 825-852). Cape Town: Oxford University Press.

Thompson, G. R., \& Bastos, A. D. S. (2004). Foot-and-mouth disease. In J. A. W. Coetzer \& R. C. Tustin (Eds.), Infectious Diseases of Livestock (pp. 1324-1365). Oxford University Press Southern Africa, Cape Town.

Valarcher, J. F., Knowles, N. J., Ferris, N. P., Paton, D. J., Zakharov, V., Sherbakov, A., ... Rasool. T. J. (2005). Recent spread of FMD virus serotype Asia 1. Veterinary Records, 157, 30. https://doi.org/10.1136/ vr.157.1.30 
Vosloo, W., Bastos, A. D. S., Sangare, O., Hargreaves, S. K., \& Thomson, G. R. (2002). Review of the status and control of foot and mouth disease in sub-Saharan Africa. Revue Scientifique et Technique de l'Office International des Epizooties, 21, 437-449. http://doi.org/10.20506/rst.21.3.1349

Vosloo, W., Bastos, A. D. S., Sahle, M., Sangare, O., \& Dwarka, R. M. (2005). Virus topotypes and the role of wildlife in foot and mouth disease in Africa. In S. A. Osofsky (Ed.), Conservation and Development Interventions at the Wildlife/Live- stock Interface: Implications for Wildlife, Livestock and Human Health (Chapter 10, pp. 67-73). Cambridge, UK: IUCN Publications Services Unit.

Wekesa, S. N., Muwanika, V. B., Siegismund, H. R., Sangula, N. A., Dhikusooka, D. T., Tjørnehøj, K., ... Belsham, G. J. (2013). Analysis of Recent Serotype O Foot-and-Mouth Disease Viruses from Livestock in Kenya: Evidence of Four Independently Evolving Lineages. Transboundary and Emerging Diseases, 62(3), 305-14. https//doi.org/10.1111/tbed.12152

Wekesa, S. N., Sangula, A. K., Belsham, G. J., Muwanika, V. B., Heller, R., Balinda, S. N., ... Siegismund, H. R. (2014). Genetic diversity of serotype A foot-and-mouth disease viruses in Kenya from 1964 to 2013; implications for control strategies in eastern Africa. Infectious Genetic Evolution, 21, 408-417. https//doi.org/10.1016/j.meegid.2013.12.006

Woodsbury, R. L. (1995). A review of the possible mechanisms for the persistence of foot-and-mouth diseases virus. Journal of Epidemiology and Infections, 114, 1-13. https//doi.org/10.1017/s0950268800051864

WRLFMD. (n.d.). Foot-and-mouth disease. Retrieved from http://www.wrlfmd.org/foot-and-mouth-disease

\section{Copyrights}

Copyright for this article is retained by the author(s), with first publication rights granted to the journal.

This is an open-access article distributed under the terms and conditions of the Creative Commons Attribution license (http://creativecommons.org/licenses/by/4.0/). 